\title{
ARGUMENTS INDICATING THAT AP AND Am STARS ARE EVOLVED SPECTROSCOPIC BINARIES
}

\author{
E.P.J. VAN DEN HEUVEL \\ (Sterrenwacht 'Sonnenborgh', Utrecht, The Netherlands, and Sterrenkundig Instituut \\ der Vrije Universiteit, Brussels, Belgium)
}

\section{Introduction}

Roughly three types of evolution of spectroscopic binaries may occur $(\mathbf{1}, \mathbf{2}, \mathbf{3}, \mathbf{4}, \mathbf{5}$, 6):

Case (a): the primary fills its Roche limit already before the end of core hydrogen burning;

Case (b): the primary fills its Roche limit after core hydrogen exhaustion but before the onset of helium burning, and

Case (c): the primary does not fill its Roche limit before the end of core helium burning.

Since long-period variables of Population I as well as Population II may reach radii of $10 \mathrm{AU}(7)$, case (c) will occur in systems with periods up to 100 years. Since about $50 \%$ of the A and B stars are spectroscopic binaries (8), many evolved spectroscopic binaries are expected to occur in the galactic system. Computations (9) show that, using the mass-ratio distribution of unevolved systems derived by Kuiper (10), the number of systems with one evolved component is expected to be at least as large as the number of unevolved systems, in the spectral regions A and B.

\section{Expected Characteristics of Evolved Spectroscopic Binaries}

When the primary component has passed through all nuclear burning stages and has become a white dwarf, the remaining secondary will be expected to show the characteristics summarised in Table 1:

(1) Due to its increase in mass (up to $87 \%$ of the mass of the primary may be transferred towards the secondary (2)), the star will, in the Hertzsprung-Russell diagram, have moved upwards along the main sequence (Figure 1). In clusters it may have become (one of) the bluest and/or brightest main-sequence star(s), beyond the turn-off point (11). Since only little of its total amount of hydrogen has been burned at that moment, the new primary will be close to the Zero Age Main Sequence (ZAMS).

(2) Table 2 (after Hack (12)) lists some of the many eclipsing binaries in which at

Perek (ed.), Highlights of Astronomy, 420-431. (1) I.A.U. 


\section{Table 1}

Expected characteristics of evolved spectroscopic binaries compared with observed characteristics of Ap and Am stars

Evolved spectroscopic binaries, expected

(1) In clusters: close to the ZAMS and near or beyond the turn-off point

(2) Slow rotation

(3) Abundance anomalies of heavy elements and iron peak, except in case (b) if $M_{\text {prim }}$ smaller than $2 \cdot 8 M_{\odot}$
Ap, Am stars, observed

(1) In clusters: close to the ZAMS and near or beyond the turn-off point

(2) Slow rotation

(3) Abundance anomalies of heavy elements and iron peak

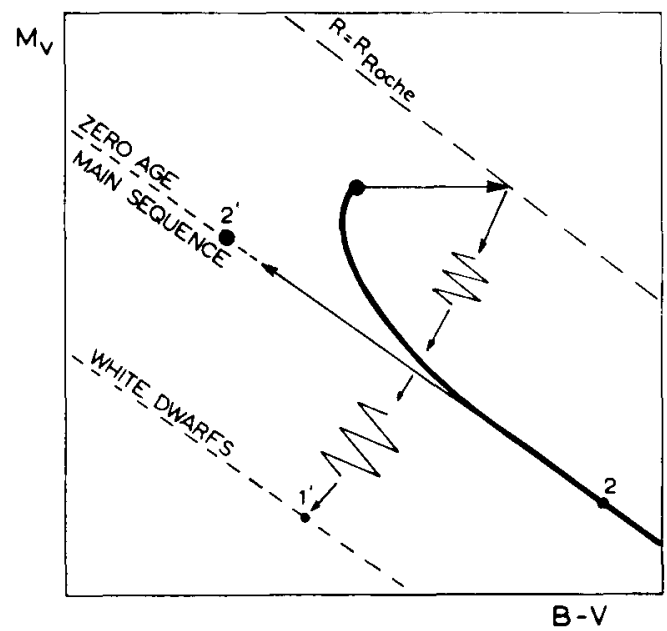

Fig. 1. The expected positions of the components of an evolved spectroscopic binary in the colourmagnitude diagram of a cluster. The heavily drawn curve indicates the cluster main sequence. $\mathrm{R}_{\mathrm{Roche}}$ is the Roche limit of the primary before the mass exchange.

Table 2

Some of the many eclipsing systems in which the more massive star is an unevolved $A$ or B star and the less massive star is an evolved subgiant (12). Initial periods are given for three initial mass ratios

$\begin{array}{cccccccc}\text { Name } & \text { Sp } 1 & \mathrm{Sp}_{2} & P \text { (days) } & \frac{m_{2}}{m_{1}} & \boldsymbol{P}_{0}\left(\alpha_{0}=\mathbf{0 . 5}\right) & \boldsymbol{P}_{0}\left(\alpha_{0}=0.2\right) & \boldsymbol{P}_{0}\left(\alpha_{0}=\mathbf{0 . 1}\right) \\ & & & & & & & \\ \text { U Sge } & \text { B9 } & \text { gG6 } & 3.38 & 0.30 & 1.72 & 7.05 & 33.6 \\ \text { TX UMa } & \text { B8 } & \text { gG4 } & 3.06 & 0.30 & 1.56 & 6.40 & 30.4 \\ \text { SX Hya } & \text { A3 } & \text { gK6 } & 2.90 & 0.35 & 1.87 & 7.67 & 36.5 \\ \text { ST Per } & \text { A3 } & \text { gG5 } & 2.65 & 0.18 & 2.72 & 11.30 & 53.0 \\ \text { S Cnc } & \text { A0 } & \text { gG5 } & 9.49 & 0.36 & 6.40 & 26.2 & 125.0 \\ \text { RY Per } & \text { B4 } & \text { F2 } & 6.86 & 0.20 & 1.69 & 6.94 & 33.0 \\ \text { RG Cep } & \text { A5 } & \text { gK3 } & 12.42 & 0.38 & 9.05 & 37.1 & 176.0 \\ \text { Lib } & \text { A0 } & \text { gG2 } & 2.33 & 0.44 & 2.07 & 8.50 & 40.4\end{array}$


present the more massive primary is an unevolved A or B star, while the less massive secondary is a probably evolved subgiant, filling its Roche limit. In these systems the mass exchange has probably already taken place. Often the unevolved A or B stars in close systems are observed to rotate slowly (13); for $P<10^{\mathrm{d}}$ the rotation is often (and for $P<4^{\mathrm{d}}$ in practically all cases) synchronised with the orbital motion (14). Computations (15) show that in systems with $P>10^{\mathrm{d}}$, large mass exchange may also slow down the rotation of the unevolved star (exchange of small amounts of mass may accelerate the rotation of the surface layers, as is probably observed in systems like $\mathrm{U}$ Cep (16)). In the computations it is assumed that, at the moment of accretion on the A or B star, the exchanged matter moves in the system with velocities synchronised with the orbital motion of the components. It is found that for rigid-body rotation as well as for conservation of angular momentum in each shell in the stellar interior, the final angular velocity of rotation will not exceed $6.8 \times 10^{-5} \mathrm{sec}^{-1}$, the average being less than $4.4 \times 10^{-5} \mathrm{sec}^{-1}$. For A0 stars these angular velocities correspond to $v_{\mathrm{e}} \approx 85 \mathrm{~km} / \mathrm{sec}$ and $57 \mathrm{~km} / \mathrm{sec}$, respectively.

(3) Except in case (b), with primary mass below $2.8 M_{\odot}(2)$, heavy-element synthesis and mass ejection may be expected during the last stages of evolution of the primary. Especially, since after the mass exchange the primary has become an evolved star of low mass, one expects that a degenerate core will develop, and flash stages like the helium and carbon flash will occur. During flashes many neutrons will be produced and the conditions will be favourable for heavy-element synthesis in the way outlined by Fowler et al. (18). Hence, when the primary has become a white dwarf, the surface layers of the remaining A or B star may be covered with the ejected heavy elements. Since in case (b) a primary less massive than $2.8 M_{\odot}$ directly evolves into a pure helium white dwarf (2), also some slowly rotating blue stragglers without abundance anomalies may be expected in A clusters.

\section{The Observed Characteristics of Ap and Am Stars}

These are summarised in the right-hand part of Table 1:

(1) As Figure 2a-2g show, in five galactic clusters the bluest and/or brightest stars are Ap or Am, while in all galactic clusters Ap and Am stars scatter about the blue edge of the top of the cluster main sequence. Since Ap and Am stars have masses $(19,20,21)$ and surface gravities $(22,23)$ similar to those of stars on the main sequence that have the same B-V, these bluest stars are probably also the heaviest cluster stars.

(2) The rotation of Ap and Am stars is intrinsically slow (24, 25, 26, 27, 28); $v_{\mathrm{e}} \sin i \approx 40 \mathrm{~km} / \mathrm{sec}$ for Am stars and $\approx 50 \mathrm{~km} / \mathrm{sec}$ for Ap stars.

(3) Ap and Am stars show probably real overabundances of heavy elements and the iron peak $(29,30)$ and underabundances of light elements like $\mathrm{He}, \mathrm{C}$ and $\mathrm{O}$.

Since in clusters like the Hyades and Coma at least 20 to $30 \%$ of the stars are spectroscopic binaries (31), one certainly expects to find a number of evolved spectro- 


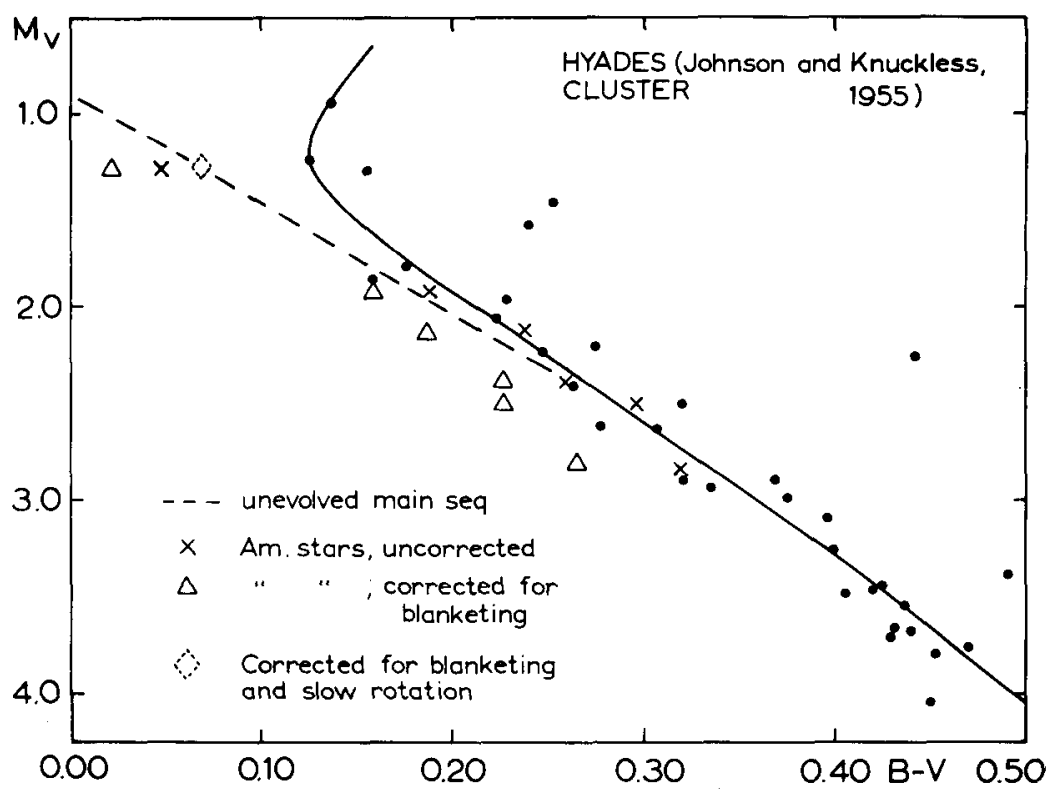

Fig. 2a. The positions of Ap and/or Am stars in the colour magnitude diagrams of seven galactic clusters (borrowed from sources (40), (41), (42), (43), (44)).

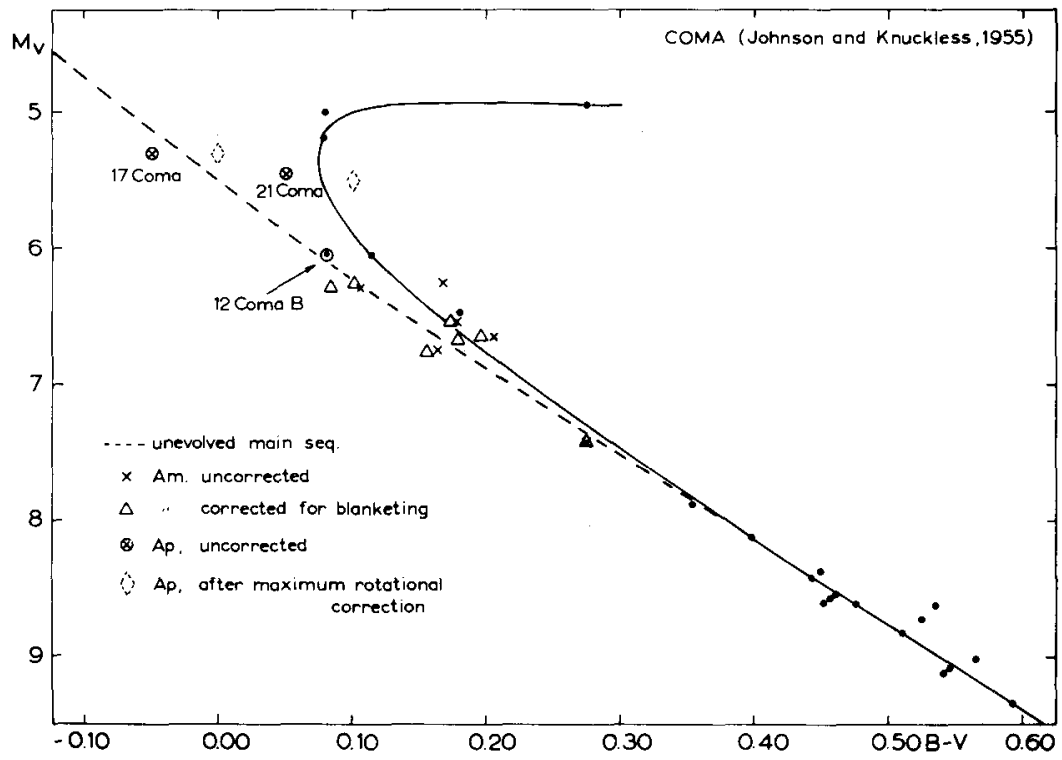

FiG. 2b. 


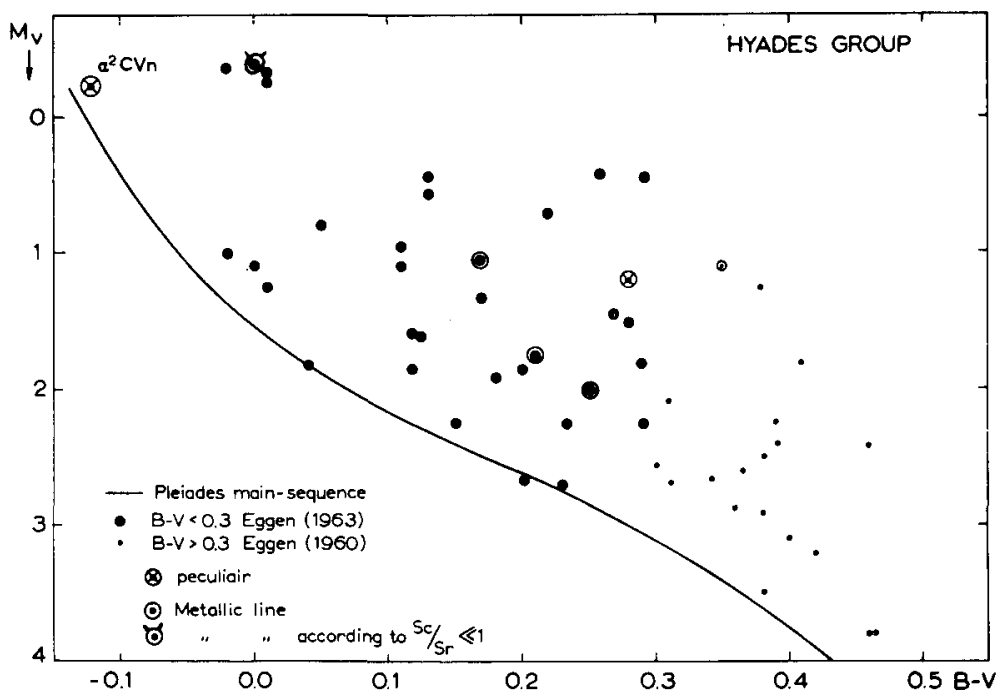

FIG. 2c.

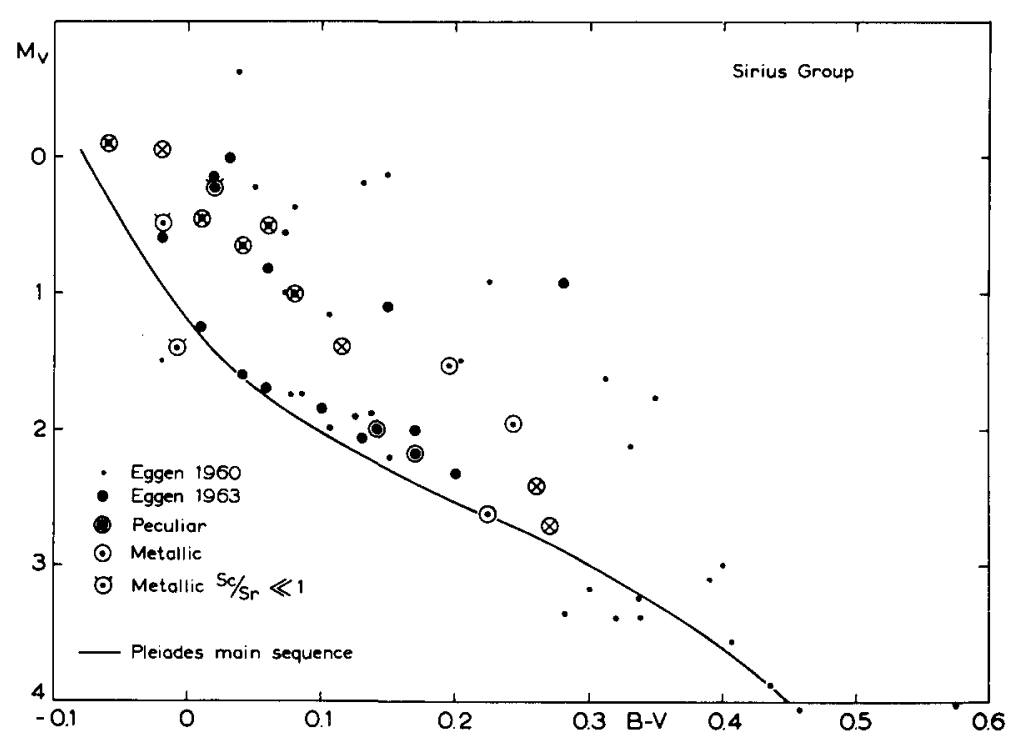

FIG. 2d.

scopic binaries in such clusters. Ap and Am stars are the only stars in these clusters which show the three above-mentioned expected characteristics of evolved spectroscopic binaries. For this reason we suggest that Ap and Am stars are spectroscopic binaries with an evolved companion (as was first suggested by Renson (32)). 


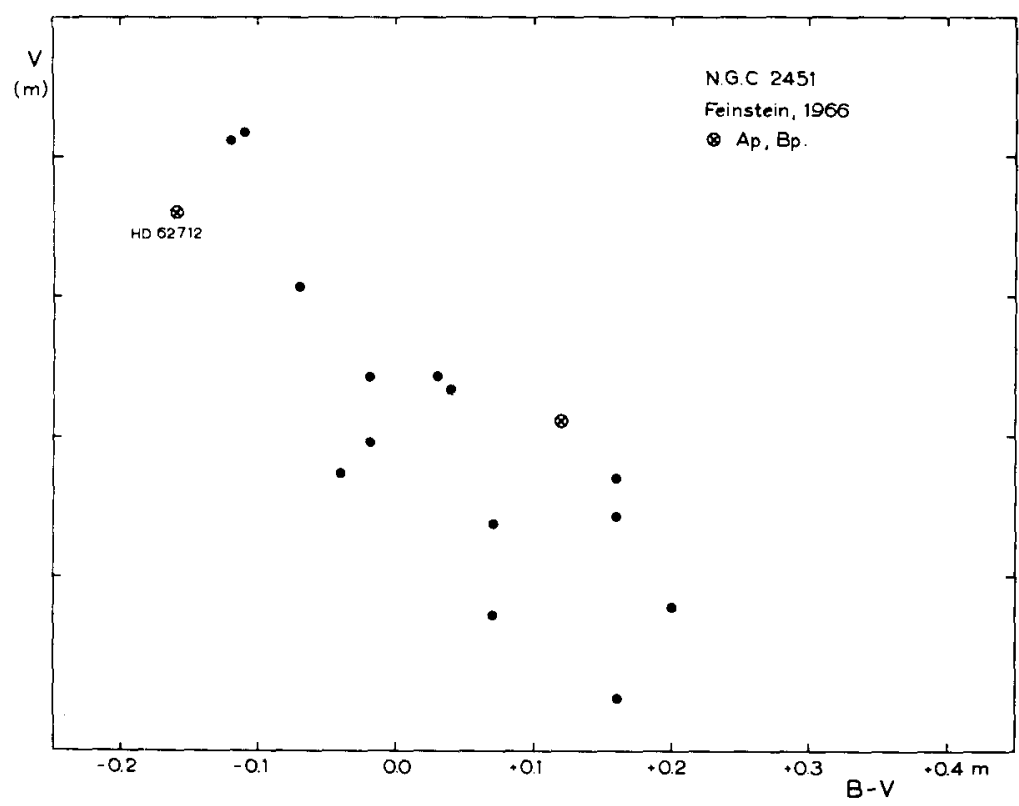

FIG. 2e.

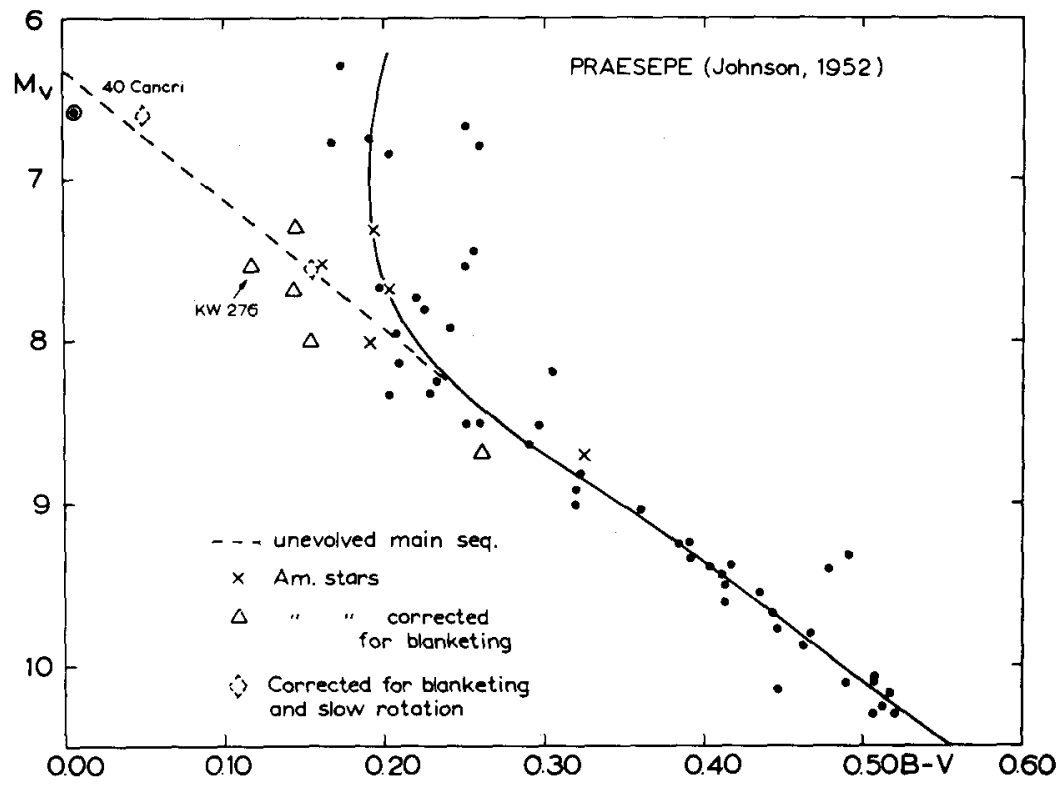

FIG. 2f. 


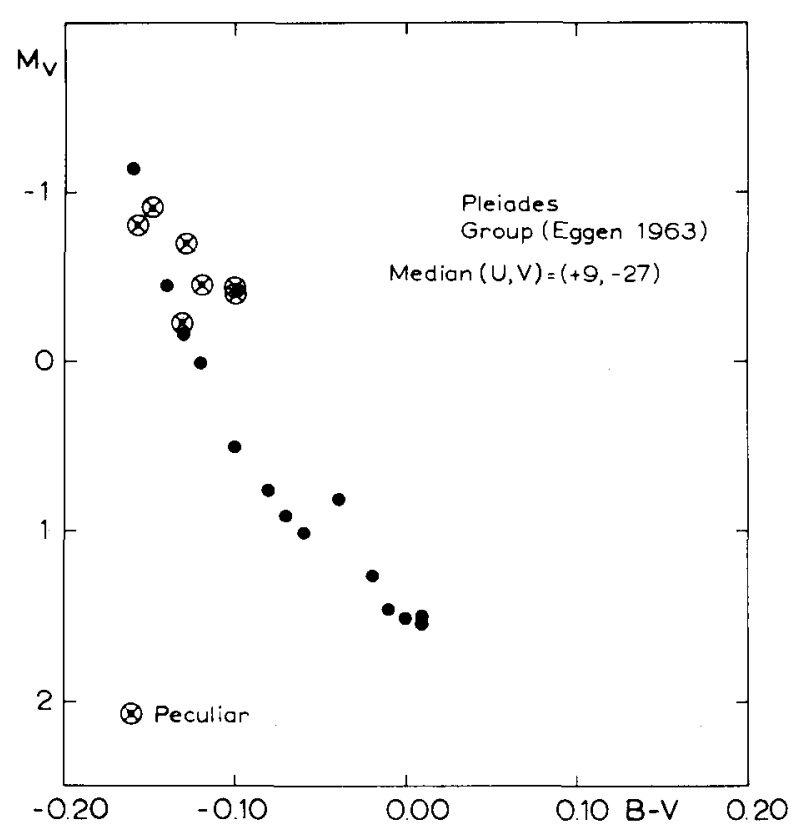

Fig. $2 \mathrm{~g}$.

\section{Possible Reasons for the Observed Different Percentages of Spectroscopic Binaries among Ap and Am Stars}

From conservation of orbital angular momentum (33) the change in period as a consequence of mass exchange is for circular orbits given by

$$
P^{\prime} / P_{0}=\left(\frac{\alpha_{0}}{\left(1+\alpha_{0}\right)^{2}} \cdot \frac{\left(1+\alpha^{\prime}\right)^{2}}{\alpha^{\prime}}\right)^{3},
$$

where $\alpha_{0}$ denotes the mass ratio of secondary and primary before the exchange and $\alpha^{\prime}$ the mass ratio of new secondary and new primary after the exchange (both are smaller than 1). If $\alpha_{0}>\alpha^{\prime}$, the final period $P^{\prime}$ is larger than $P_{0}$. If $\alpha_{0}<\alpha^{\prime}$ then $P^{\prime}$ is smaller than $P_{0}$ (see Figure 3). Assuming the final white dwarf to have a mass of $0.7 M_{\odot}$ (which is the average for white dwarfs on the high-mass sequence (34)) and the original primary mass to be $M$, the final mass ratio $\alpha^{\prime}$ is equal to

$$
\alpha^{\prime}=\frac{0.7}{\left(\alpha_{0}+1\right) M-0.7}
$$

and

$$
P^{\prime} / P_{0}=\left[\frac{\alpha_{0}(M)^{2}}{\left(\left(\alpha_{0}+1\right) M-0 \cdot 7\right) 0 \cdot 7}\right]^{3} .
$$




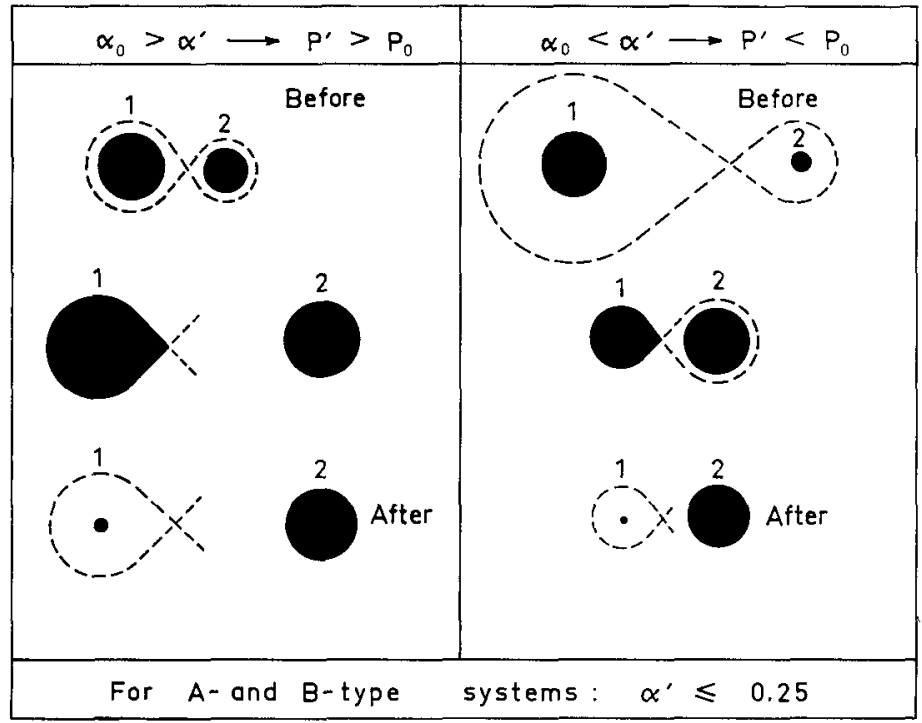

FIG. 3. The change in separation as a consequence of mass exchange, as a function of the original and final mass ratios $\alpha_{0}$ and $\alpha^{\prime}$.

Consequently, if the period is increased by a factor $a$ due to the exchange, $\alpha_{0}$ must have been equal to

$$
\alpha_{0}(a, M)=\frac{a^{1 / 3}(0.7 M-0 \cdot 49)}{M^{2}-0.7 a^{1 / 3} M} .
$$

Assuming the mass-ratio distribution derived by Kuiper (10) and by Van Albada (personal communication)

$$
\varphi\left(\alpha_{0}\right)=2 /\left(1+\alpha_{0}\right)^{2},
$$

the percentage of spectroscopic binaries of which the period increases by a factor $a$ or more is equal to

$$
Q_{M}(a)=\int_{\alpha_{0}(a, M)}^{1} \frac{2}{\left(1+\alpha_{0}\right)^{2}} \mathrm{~d} \alpha_{0} .
$$

Equation (6) yields $Q_{1 \cdot 5}(1)=0.36 ; Q_{2}(1)=0.50$ and $Q_{4}(1)=0.75$. Hence, for A5-F2 stars $\left(M=1 \cdot 5-2 M_{\odot}\right)$, only in $36 \%$ to $50 \%$ of the cases the orbit will be increased, while for $M=4 M_{\odot}(\mathrm{B} 7-\mathrm{A} 0)$ in $75 \%$ of the cases the period will be increased after the exchange. Since furthermore a $0.7 M_{\odot}$ white-dwarf companion, in the same orbit, will induce two times larger variations in radial velocity in a $2 M_{\odot}$ primary than in a $4 M_{\odot}$ primary, the observable percentage of spectroscopic binaries among B8p stars is expected to be much lower than among Am stars (which in general have 


\section{Table 3}

\section{The distribution of the periods of B-type spectroscopic binaries in Moore and Neubauer's (35) catalogue}

$\begin{array}{ccc}P(\mathrm{~d}) & \text { B0-B5 } & \text { B6-B9.5 } \\ <1^{\mathrm{d}} & 1.2 \% & 2.9 \% \\ 1-1.5 & 8 \cdot 1 & 2.9 \\ 1.5-2 & 7 \cdot 0 & 8.6 \\ 2-3 & 20.9 & 11 \cdot 4 \\ 3-9 & 32.5 & 40.0 \\ 9-27 & 15 \cdot 1 & 17.1 \\ 27-81 & 7 \cdot 0 & 11.4 \\ 81-243 & 7.0 & 5.7 \\ >243 & 1.2 & \end{array}$

spectral type later than A3). With the distribution of periods of normal B-type spectroscopic binaries (Table 3, after Moore and Neubauer (35)) one computes that after the mass exchange only $35 \%$ of the stars with a mass of $4 M_{\odot}$ will show variations in radial velocity larger than $20 \mathrm{~km} / \mathrm{sec}$, while $65 \%$ of the stars of $2 M_{\odot}$ mass will show such variations in radial velocity. Since only $6 \%$ of the spectroscopic binaries in Moore and Neubauer's catalogue show variations in radial velocity below $20 \mathrm{~km} / \mathrm{sec}$, a velocity variation of $20 \mathrm{~km} / \mathrm{sec}$ is roughly at the limit of detectability for the resolutions used for radial velocity catalogues. In this way, or by the presence of a less massive white-dwarf companion for Ap stars than for Am stars, one may explain the observed apparently low percentage of spectroscopic binaries among Ap stars (36) and the high percentage among Am stars (24). In fact we expect all Ap stars to be binaries, which, however, will only be recognised as such if sufficiently high spectral resolutions are used.

\section{Suggested Reason for the Difference between Ap and Am Stars}

The solid curve in Figure 4 represents the lower limiting binary period for case (b) evolution computed from Iben's results for $\alpha_{0}=0.5$ (this means: systems below the solid line are in case (b)). For other $\alpha_{0}$-values the limiting periods differ by less than $15 \%$ from these values. The periods of the bulk of the normal spectroscopic binaries are also indicated. Since most B-type systems have $3^{\mathrm{d}} \leqslant P \leqslant 9^{\mathrm{d}}(\mathbf{3 5})$ and practically all normal A5 to F2V spectroscopic binaries have $P>100^{\mathrm{d}}$ (37), most B-type spectroscopic binaries are in case (b) while most later A-type systems are in case (c). Case (a) is relatively unimportant since it occurs only for B-type systems with $P<2^{d}$ and for A-type systems with $P<1^{\text {d }}$. Since also the transition from the dominance of Ap stars to the dominance of Am stars (among slow rotators with enhanced metal spectrum) occurs in the early A spectral region, we suggest that Ap stars are the products of case (b) evolution of systems with original primary mass above $2 \cdot 8 M_{\odot}$, while Am stars are 


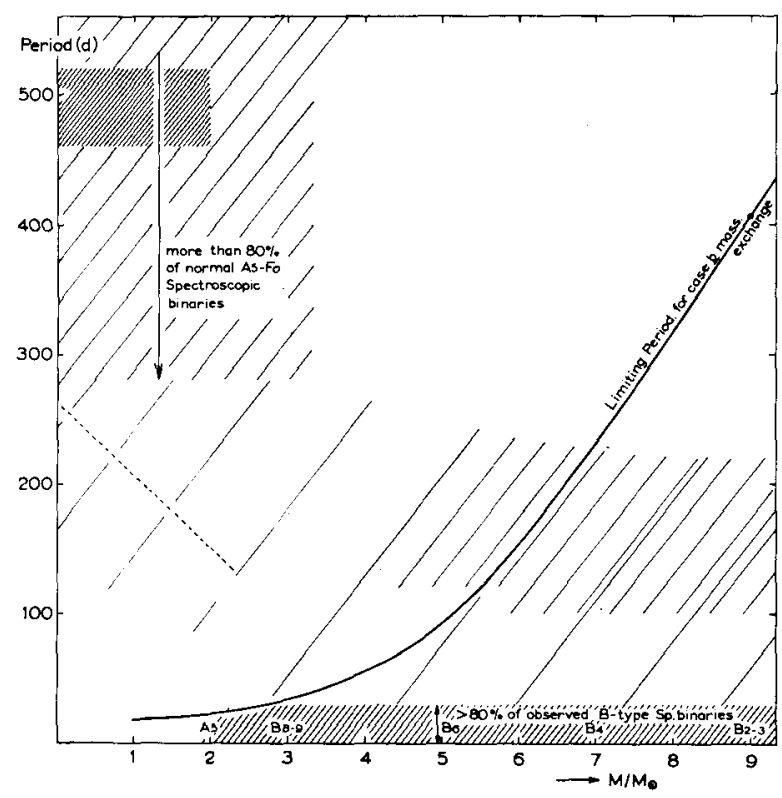

FIG. 4. The limiting binary period for case ( $b$ ) mass exchange as a function of the original primary mass, for an original mass ratio of 0.5 . Systems below the heavy curve are in case (b). The heavily shaded areas indicate the periods of the bulk of the normal A-and B-type spectroscopic binaries. The dashed curve indicates the limiting period when the occurrence of the helium flash is taken into account.

the products of case (c) evolution. Since primaries less massive than $2 \cdot 8 M_{\odot}$ will, by case (b) evolution, directly evolve into pure-helium white dwarfs (2), no Ap stars are expected in clusters in which unevolved stars near the turn-off point are less massive than $2.8 M_{\odot}$. In these clusters only Am stars or slowly rotating blue stragglers without abundance anomalies - like 40 Cancri in Praesepe (38)-are expected to be found. On the other hand, Am stars are expected, in principle, in any cluster, but will be rare in B-clusters (since most B stars are in case (b)). Indeed, some low Sc/Sr Am stars occur already in the Pleiades (39)).

\section{References}

1. Kippenhahn, R., Weigert, A. (1967) Z. Astrophys., 65, 251.

2. Kippenhahn, R., Kohl, K., Weigert, A. (1967) Z. Astrophys., 66, 58.

3. Paczyński, B. (1966) Acta astr., 16, 231.

4. Paczyński, B. (1967) Acta astr., 17, 1.

5. Paczyński, B., Ziolkowski, J. (1967) Acta astr., 17, 7.

6. Plavec, M. (1967) On the evolution of double stars, ed. J. Dommanget (Obs. Royal, Uccles, Belgium), p. 83.

7. Smak, J. (1964) Astrophys. J., Suppl. 11, 141.

8. Batten, A. (1967) A. Rev. Astr. Astrophys., 5, 25. 
9. Van den Heuvel, E.P.J. (1968) Bull. astr. Inst. Netherl., 19, 326.

10. Kuiper, G.P. (1935) Publ. astr. Soc. Pacific, 71, 451.

11. McCrea, W.H. (1964) Mon. Not. R. astr. Soc., 128, 147.

12. Hack, M. (1963) in Star Evolution, Ed. by P. Gratton, Acadenic Press, New York, p. 452.

13. Huang, Su-Shu (1966) A. Rev. Astr. Astrophys., 4, 35.

14. Plaut, L. (1959) Publ. astr. Soc. Pacific, 71, 167.

15. Van den Heuvel, E.P.J. (1968) Bull. astr. Inst. Netherl. (in press).

16. Struve, O. (1963) Publ. astr. Soc, Pacific, 75, 207.

17. Struve, O. (1946) Astrophys. J., 103, 76.

18. Fowler, W.A., Burbidge, E. M., Burbidge, G. R., Hoyle, F. (1965) Astrophys. J., 142, 423.

19. Eggen, O.J. (1965) A. Rev. astr. Astrophys., 3, 235.

20. Abt, H.A., Conti, P.S., Deutsch, A.J., Wallerstein, G. (1966) Paper presented at the 122nd Meeting of the AAS.

21. Hyland, A. R. (1967) Proc. astr. Soc. Australia, 1, 14.

22. Baschek, B., Oke, J.B. (1965) Astrophys. J., 141, 1404.

23. Van 't Veer-Menneret, C. (1966) in IAU Symp. 26, Ed. by H. Hubenet, Academic Press, New York, p. 261.

24. Abt, H.A. (1961) Astrophys. J., Suppl. 6, 37.

25. Kraft, R.P. (1965) Astrophys. J., 142, 703.

26. Sargent, W.L.W., Strittmatter, P.A. (1966) Astrophys. J., 145, 938.

27. Van den Heuvel, E.P.J. (1967) Bull. astr. Inst. Netherl., 19, 11.

28. Schöneich, W. (1967) Astr. Nachr., 61, 290.

29. Sargent, W.L.W. (1966) in IAU Symp. 26, Ed. by H. Hubenet, Academic Press, New York, p. 247.

30. Conti, P.S. (1965) Astrophys. J., 142, 1594.

31. Kraft, R.P. (1965) Astrophys. J., 142, 681.

32. Renson, P. (1965) Ann. Astrophys., 28, 679.

33. Huang, Su-Shu (1963) Astrophys. J., 138, 471.

34. Eggen, O.J., Greenstein, J.L. (1965) Astrophys. J., 141, 83.

35. Moore, J.H., Neubauer, F.J. (1948) Lick Obs. Bull., 20, 1.

36. Jaschek, C., Jaschek, M. (1958) Z. Astrophys., 45, 35.

37. Abt, H.A. (1965) Astrophys. J., Suppl. 11, 429.

38. Conti, P.S., Wallerstein, G., Wing, F. (1965) Astrophys. J., 142, 423.

39. Conti, P.S., Strom, S.E. (1967) Astrophys. J. (in press).

40. Johnson, H.L., Knuckless, C.F. (1955) Astrophys. J., 122, 209.

41. Treanor, P.J. (1960) Mon. Not. R. astr. Soc., 121, 503.

42. Eggen, O.J. (1963) Astr. J., 68, 483.

43. Eggen, O.J. (1963) Astrophys. J., Suppl. 76.

44. Feinstein, A. (1966) Publ. astr. Soc. Pacific, 78, 301.

\section{DISCUSSION}

Roxburgh: How did you determine the rotation velocities of $30 \mathrm{~km} / \mathrm{sec}$ ?

Van den Heuvel: Since A or B stars in close binaries with period of less than 10 days are observed to be slow rotators, I expect that these stars will continue to rotate slowly. This was my argument.

Mestel: I heard last year from Dr. H. Abt that he had begun a study of the proportion of Ap stars in close binary systems, and that he was finding a surprisingly high number. Is there any observational astronomer present who can confirm or deny this?

Batten: I also understood from Dr. Abt a few months ago that he was finding a high proportion of spectroscopic binaries amongst Ap stars.

Van den Heuvel: I think all Ap stars are spectroscopic binaries. Only, as shown by the formulas for the change in period, the period is probably increased while the companion has a low mass. Hence, I expect that spectroscopic detection of the presence of the companion will not be easy. 
Underhill: Could one paraphrase your remarks to read that membership in a close binary leads to slow rotation, and that slowly rotating stars, however they achieve this state, very frequently have a spectrum which is classified as being peculiar? I refrain at this time from interpreting the peculiarities in term of particular physical conditions and abundances.

Van den Heuvel: I do not think that all slow rotators should have abnormal spectra. There are slow rotators which have normal spectra, like the blue straggler $40 \mathrm{Cnc}$ in the Praesepe cluster. It has a normal spectrum as Dr. Conti has shown. Abnormal spectra occur in my opinion only in evolved spectroscopic binaries in which nucleosynthesis has taken place. 